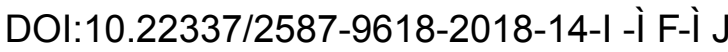

\title{
THE GENERALIZED BIFRACTIONAL BROWNIAN MOTION
}

\author{
Charles El-Nouty \\ LAGA, Université Paris XIII, Sorbonne Paris Cité, FRANCE
}

\begin{abstract}
To extend several known centered Gaussian processes, we introduce a new centered Gaussian process, named the generalized bifractional Brownian motion. This process depends on several parameters, namely $\alpha>0, \beta>0,0<H<1$ and $0<K \leq 1$. When $K=1$, we investigate its convexity properties. Then, when $2 H K \leq 1$, we prove that this process is an element of the QHASI class, a class of centered Gaussian processes, which was introduced in 2015.
\end{abstract}

Keywords: convexity, quasi-helix, approximately stationary increments

\section{ОБОБЩЕННОЕ БИФРАКТАЛЬНОЕ БРОУНОВСКОЕ ДВИЖЕНИЕ}

\author{
ШІ. Эль-Нути \\ Университет Париж ХІІІ, Сорбонна - Париж - Сите, ФРАНЦИЯ
}

\begin{abstract}
Аннотация: Расширение нескольких центрированных гауссовский процессов требует введения нового процесса, названного бифрактальным броуновским движением. Этот процесс зависит от нескольких параметров, а именно: $\alpha>0, \beta>0,0<H<1$ и $0<K \leq 1$. Для случая, когда параметр $K=1$, исследуется свойство выпуклости. Для случая, когда $2 H K \leq 1$, доказывается принадлежность этого процесса к квази-классу (обладанием квази-канонической кривой постоянного склона), и к классу центральных гауссовских процессов.
\end{abstract}

Ключевые слова: выпуклость, квази-каноническая кривая постоянного склона, приближенно стационарные прирощении

\section{INTRODUCTION}

Let $\left\{B_{H, K}(t), t \in \mathrm{R}\right\}$ be a bifractional Brownian motion (bBm) with indices $0<H<1$ and $0<K \leq 1$, i.e. a centered Gaussian process such that $B_{H, K}(0)=0$, with probability 1 , and

$$
\begin{aligned}
& \mathrm{E}\left(B_{H, K}(t) B_{H, K}(s)\right) \\
& =\frac{1}{2^{K}}\left(\left(|t|^{2 H}+|s|^{2 H}\right)^{K}-|t-s|^{2 H K}\right) .
\end{aligned}
$$

We can verify that

$$
\operatorname{Var} B_{H, K}(t)=|t|^{2 H K}
$$

and that the $\mathrm{bBm}$ is self-similar with index $H K$. Note also that the process $B_{H, 1}$ is the fractional Brownian motion (fBm) and therefore the process $B_{1 / 2,1}$ is the ordinary Wiener process. Straightforward computations show $B_{H, K}$ has no stationary increment. However, the $\mathrm{bBm}$ is a HK-quasi-helix in the sense of Kahane ([1], p. 137) and its increments are approximately stationary for small increments. Houdré and Villa [2] introduced the $\mathrm{bBm}$ and established the previous results.

Consider the following centered Gaussian process $Y:=Y_{\alpha, \beta, H, K}$ defined as follows: 


$$
\begin{aligned}
Y(t) & :=Y_{\alpha, \beta, H, K}(t) \\
& =\alpha B_{H, K}(t)+\beta B_{H, K}(-t)
\end{aligned}
$$

with $t \geq 0, \alpha>0, \beta>0$. Set

$$
\alpha(K)=1 /\left(2^{(2-K) / 2}\right), 0<K \leq 1 .
$$

The introduction of the process $Y$ is motivated by the fact that this process was already introduced for specific values of $\alpha, \beta$ and $K$. Indeed, the process $Y_{\alpha(1), \alpha(1), H, 1}$ was introduced in [3] and was named the sub-fractional Brownian motion. El-Nouty and Journé [4] extended the former process by introducing the process $Y_{\alpha(K), \alpha(K), H, K}$, which was named the subbifractional Brownian process ( $\mathrm{sbBm})$. Finally, Zili [5] introduced the process $Y_{\alpha, \beta, H, 1}$, which was named the generalized fractional Brownian motion $(\mathrm{gfBm})$. This is why we will name $Y$ the generalized fractional Brownian motion ( $\mathrm{gbBm})$. Set for $s, t \geq 0$

$$
\begin{aligned}
& \sigma^{2}(s, t):=\sigma_{\alpha, \beta, H, K}^{2}(s, t) \\
& =\mathrm{E}\left(\left(Y_{\alpha, \beta, H, K}(t)-Y_{\alpha, \beta, H, K}(s)\right)^{2}\right) .
\end{aligned}
$$

Let us study the convexity properties of

$$
\sigma^{2}(s, t):=\sigma_{\alpha, \beta, H, 1}^{2}(s, t)
$$

on the set

$$
T=\left\{(s, t) \in[0,1]^{2}: s \leq t\right\} .
$$

Our first result is stated in the following proposition.

Proposition 1. I. If $H \geq 1 / 2$, then the function $\sigma_{\alpha, \beta, H, 1}^{2}(s, t), \quad(s, t) \in T \quad$ is convex and has a unique maximum at the point $(0,1)$.

II. If $H<1 / 2$, then the function $\sigma_{\alpha, \beta, H, 1}^{2}(s, t)$, $(s, t) \in T$ is concave and has a unique maximum at the point $(0,1)$.

Note the difference between the case $0<H<1 / 2$ and the case $1 / 2<H<1$, i.e. between short-range dependence and long-range dependence. This phenomenon was already observed by several authors in the $\mathrm{fBm}$ case (Beran ([6], p52), Samorodnitsky and Taqqu ([7], p. 123)). Proposition 1 establishes that the $\mathrm{fBm}$ and the gfBm are similar from the convexity point of view. However, when one compares Proposition 1 with Proposition 1.1 in [8], he can observe the difference between the $\mathrm{gfBm}$ and the $\mathrm{bBm}$. This implies that there is a significant difference between the processes $Y_{\alpha, \beta, H, 1}$ and $Y_{\alpha, \beta, H, K}$, with $K<1$.

The quasi-helix with approximately stationary increments (QHASI) class of centered Gaussian processes was introduced by El-Nouty [9] and was defined as follows. A centered Gaussian process $\quad\{X(t), t \in I \subset R\} \quad$ belongs to the QHASI class if it fulfills the five following assumptions:

- A1: $X(0)=0$ with probability 1 ,

- A2: there exists $\lambda>0$ such that $X$ is self-similar with index $\lambda$,

- A3: there exists $0<C_{1} \leq C_{2}<+\infty$ such that $\forall(s, t) \in I^{2}$

$C_{1}|t-s|^{2 \lambda} \leq \mathrm{E}(X(t)-X(s))^{2} \leq C_{2}|t-s|^{2 \lambda}$,

- A4: there exists $C_{3} \in\left[C_{1}, C_{2}\right]$ such that

$$
\begin{gathered}
\forall(s, t) \in I^{2}, t \geq s, s t \neq 0, \text { when } t-s \rightarrow 0, \\
\mathrm{E}(X(t)-X(s))^{2} \sim C_{3}(t-s)^{2 \lambda}
\end{gathered}
$$

- A5: there exists $C_{4} \in\left[C_{1}, C_{2}\right]$ such that

$$
\forall t \in I, \mathrm{E} X(t)^{2}=C_{4}|t|^{2 \lambda}
$$


Let us state three known results. The first one is due Houdré and Villa [2], the second one to [10] and the last one to El-Nouty [9].

Theorem 2. The bBM is an element of the QHASI class.

Theorem 3. The sfBm is an element of the QHASI class.

Theorem 4. The sbBm is an element of the QHASI class.

We insist on the fact that the values of $\lambda, C_{1}$, $C_{2}, C_{3}$ and $C_{4}$ for the $\mathrm{bBm}$, the sfBm and the sbBm can be found in El-Nouty [9]. Using some results of Zili [5] and introducing some additional computations, we get the following result.

Theorem 5. The gfBm is an element of the QHASI class, with

- $\lambda=H$,

- $C_{1}=\min \left(\alpha^{2}+\beta^{2},(\alpha+\beta)^{2}-2^{2 H} \alpha \beta\right)$,

- $C_{2}=\max \left(\alpha^{2}+\beta^{2},\left((\alpha+\beta)^{2}-2^{2 H} \alpha \beta\right)\right)$,

- $C_{3}=\alpha^{2}+\beta^{2}$,

- $C_{4}=\alpha^{2}+2\left(1-2^{2 H-1}\right) \alpha \beta+\beta^{2}$.

Our main result is stated in the following theorem.

Theorem 6. Assume that $2 H K \leq 1$. Then the $\mathrm{gbBm}$ is an element of the QHASI class, with

- $\lambda=H K$,

- $C_{1}=(\alpha+\beta)^{2}-2^{2-K} \alpha \beta$,

- $C_{2}=2^{1-K}\left((\alpha+\beta)^{2}-2^{2 H K} \alpha \beta\right)$,

- $C_{3}=2^{1-K}\left(\alpha^{2}+\beta^{2}\right)$,

- $C_{4}=\alpha^{2}+2\left(1-2^{2 H K-K}\right) \alpha \beta+\beta^{2}$.

Let us make some comments on the above theo- rem. When

$$
H \leq 1 / 2 \text { and } K=1 \text {, }
$$

theorem $\mathrm{TH}$ is similar to theorem tutu. When

$$
2 H K \leq 1 \text { and } \alpha=\beta=\alpha(K),
$$

theorem TH is similar to theorem JLJ. Finally, as expected, note the importance of the hyperbola

$$
2 H K=1 .
$$

This phenomenon was already observed in ElNouty [11], El-Nouty and Journé [12] and Russo and Tudor [13].

Let us investigate the case

$$
1<2 H K<2 .
$$

There is no difficulty to determine $\lambda, C_{3}$ and $C_{4}$. Indeed they have the same values as those found in the case

$$
2 H K \leq 1 .
$$

A careful reading of the proof of theorem $\mathrm{TH}$ enables to state the following lemma.

Lemma 7. Assume that $2 H K>1$. Then $\forall(s, t) \in I^{2}$

$$
\sigma^{2}(s, t) \leq 2^{1-K}\left(\alpha^{2}+\beta^{2}\right)|t-s|^{2 H K} .
$$

The question of the existence of a constant $C_{1}$ is still an open one.

In section 2, we prove Proposition 1. The proof of Theorem 6is postponed to section 3. In the sequel, there is no loss of generality in assuming $K<1$. 


\section{PROOF OF PROPOSITION 1}

Recall that $K=1$ in this section. We have for any $t \geq s$

$$
\begin{aligned}
\sigma^{2}(s, t):=\sigma_{\alpha, \beta, H, 1}{ }^{2}(s, t) \\
=s^{2 H}\left(\left(\alpha^{2}+\beta^{2}\right)(u-1)^{2 H}\right. \\
\left.\quad+2^{2 H} \alpha \beta\left(2^{1-2 H}(u+1)^{2 H}-u^{2 H}-1\right)\right) \\
:=s^{2 H} \lambda(u),
\end{aligned}
$$

where

$$
u=t / s \geq 1 .
$$

There is no difficulty to deal with the case $H=1 / 2$. When $H \neq 1 / 2$, the derivative of order 2 of the function $\lambda$ is

$$
\lambda^{(2)}(u)=2 H(2 H-1) s^{2 H} g(u),
$$

where

$$
\begin{aligned}
g(u) & =\left(\alpha^{2}+\beta^{2}\right)(u-1)^{2 H-2} \\
& +2^{2 H} \alpha \beta\left(2^{1-2 H}(u+1)^{2 H-2}-u^{2 H-2}\right) .
\end{aligned}
$$

Let us study the sign of the function $g$. We have

$$
g(u) \geq 2 \alpha \beta h(u),
$$

where

$$
h(u)=(u-1)^{2 H-2}+(u+1)^{2 H-2}-2^{2 H-1} u^{2 H-2} .
$$

Since

$$
2 H-2<0,
$$

the function $u \rightarrow u^{2 H-2}, u \geq 1$ is convex, and therefore

$$
(u-1)^{2 H-2}+(u+1)^{2 H-2} \geq 2 u^{2 H-2} .
$$

Hence,

$$
h(u)=\left(2-2^{2 H-1}\right) u^{2 H-2}>0 .
$$

Combining (2.4) and (2.3) with (2.1), we establish that, if $H>1 / 2$, then the function $\sigma_{\alpha, \beta, H, 1}^{2}(s, t), \quad(s, t) \in T$ is convex, else the function is concave.

By using (2.1), we have for any real $s, t, s \neq t$, and $a>0$

$$
\sigma^{2}(s, t)>0, \sigma^{2}(s, s)=0
$$

and

$$
\sigma^{2}(a s, a t)=a^{2 H} \sigma^{2}(s, t) .
$$

Thus, we get

$$
s \frac{\partial \sigma^{2}(s, t)}{\partial s}+t \frac{\partial \sigma^{2}(s, t)}{\partial t}=2 H \sigma^{2}(s, t) .
$$

If

$$
\frac{\partial \sigma^{2}(s, t)}{\partial s}=\frac{\partial \sigma^{2}(s, t)}{\partial t}=0,
$$

then (2.5) yields that

$$
\sigma^{2}(s, t)=0
$$

and consequently

$$
s=t .
$$

Thus, there is no maximum of $\sigma^{2}(s, t)$ in the interior of $T$.

Let us investigate the existence of a maximum of $\sigma^{2}(s, t)$ on the border of $T$. Note that

$$
\sigma^{2}(0, t)=\left(\alpha^{2}+\left(2-2^{2 H}\right) \alpha \beta+\beta^{2}\right) t^{2 H}
$$

has a unique maximum at the point $t=1$. Thus, 
we have to study the function

$$
\begin{aligned}
& \sigma^{2}(s, 1)=\left(\alpha^{2}+\beta^{2}\right)(1-s)^{2 H} \\
& \quad+2^{2 H} \alpha \beta\left(2^{1-2 H}(1+s)^{2 H}-s^{2 H}-1\right) .
\end{aligned}
$$

We have by differentiation

$$
\begin{aligned}
& \frac{d \sigma^{2}(s, 1)}{d s}=2 H\left(-\left(\alpha^{2}+\beta^{2}\right)(1-s)^{2 H-1}\right. \\
& \left.+2^{2 H} \alpha \beta\left(2^{1-2 H}(1+s)^{2 H-1}-s^{2 H-1}\right)\right) .
\end{aligned}
$$

We must consider the following three cases:

Case $1 . H=1 / 2$.

Since

$$
\frac{d \sigma^{2}(s, 1)}{d s}=-\left(\alpha^{2}+\beta^{2}\right)<0,
$$

the function $\sigma^{2}(s, t),(s, t) \in T$ has a unique maximum at the point $(0,1)$. Using (2.6), we have

$$
\sigma^{2}(0,1)=\alpha^{2}+\beta^{2}
$$

Case 2. $2 H>1$.

We have

$$
\begin{aligned}
\frac{d \sigma^{2}(s, 1)}{d s} \leq 0 \Leftrightarrow & \\
2 \alpha \beta(1+s)^{2 H-1} \leq & \left(\alpha^{2}+\beta^{2}\right)(1-s)^{2 H-1} \\
& +2 \alpha \beta(2 s)^{2 H-1} .
\end{aligned}
$$

Recall that

$$
2 \alpha \beta \leq \alpha^{2}+\beta^{2} .
$$

To prove (2.7), it suffices to verify

$$
(1+s)^{2 H-1} \leq(1-s)^{2 H-1}+(2 s)^{2 H-1} .
$$

Inequality (2.8) is true at the points 0 and 1 . Set

$$
u=1 / s \geq 1 \text {. }
$$

Thus, inequality (2.8) can be rewritten as follows:

$$
(u+1)^{2 H-1} \leq(u-1)^{2 H-1}+2^{2 H-1} .
$$

Set

$$
g(u)=(u+1)^{2 H-1}-(u-1)^{2 H-1}-2^{2 H-1} .
$$

We have

$$
g^{\prime}(u)=(2 H-1)\left((u+1)^{2 H-2}-(u-1)^{2 H-2}\right) \leq 0 .
$$

Since

$$
g(1)=0,
$$

we prove that $g \leq 0$ and consequently inequality (2.9).

The function $\sigma^{2}(s, t),(s, t) \in T$ has a unique maximum at the point $(0,1)$. Using (2.6), we have

$$
\sigma^{2}(0,1)=\alpha^{2}+\left(2-2^{2 H}\right) \alpha \beta+\beta^{2} .
$$

Case 3. $2 H<1$.

To show that

$$
\frac{d \sigma^{2}(s, 1)}{d s} \leq 0
$$

it suffices to establish that 


$$
\begin{gathered}
2^{1-2 H}(1+s)^{2 H-1}-s^{2 H-1} \leq 0 \\
\Leftrightarrow(1+s)^{2 H-1} \leq(2 s)^{2 H-1} .
\end{gathered}
$$

Since

$$
2 H-1<0 \text { and } s \leq 1 \text {, }
$$

the result is true. Therefore, the function $\sigma^{2}(s, t),(s, t) \in T$ has a unique maximum at the point $(0,1)$. Using (2.6), we have

$$
\sigma^{2}(0,1)=\alpha^{2}+\left(2-2^{2 H}\right) \alpha \beta+\beta^{2}
$$

The proof of Proposition 1 is complete.

\section{PROOF OF THEOREM 6}

We can easily remark that the process $Y$ is a centered Gaussian process such that $Y(0)=0$ with probability 1 and $Y$ is self-similar with index $H K$. The covariance function of the process $Y$ is given in the following lemma.

Lemma 8. We have for $t \geq s \geq 0$

$$
\begin{aligned}
\mathrm{E}(Y(t) Y(s)) & =\frac{1}{2^{K}}\left((\alpha+\beta)^{2}\left(t^{2 H}+s^{2 H}\right)^{K}\right. \\
& -\left(\alpha^{2}+\beta^{2}\right)(t-s)^{2 H K} \\
& \left.-2 \alpha \beta(t+s)^{2 H K}\right)
\end{aligned}
$$

and therefore

$$
\mathrm{E}\left(Y(t)^{2}\right)=\left(\alpha^{2}+2\left(1-2^{2 H K-K}\right) \alpha \beta+\beta^{2}\right) t^{2 H K}
$$

Proof. It suffices to combine (1.1) with (1.2).

Remark 9. Lemma 8 gives the value of the constant $C_{4}$.

Set for $t \geq s \geq 0$

$$
\begin{aligned}
F_{H, K}(s, t)= & 2\left(\frac{t^{2 H}+s^{2 H}}{2}\right)^{K} \\
- & t^{2 H K}-s^{2 H K} \geq 0, \\
F_{\frac{1}{2}, 2 H K}(s, t) & =2\left(\frac{t+s}{2}\right)^{2 H K} \\
& -t^{2 H K}-s^{2 H K}
\end{aligned}
$$

The functions given in (3.1) and (3.2) will play a key role in the proofs of our results. Let us recall the following basic proposition.

Proposition 10. When $0<2 H K<1$, $F_{1 / 2,2 H K} \geq 0$. When $2 H K=1, F_{1 / 2,1}=0$. When $1<2 H K<2, F_{1 / 2,2 H K} \leq 0$.

Remark 9. When $2 H K \leq 1$, the function $F_{\frac{1}{2}, 2 H K}$ can be viewed as $F_{H, K}$ with $H=\frac{1}{2}$.

We can state the second technical lemma.

Lemma 12. We have for $t \geq s \geq 0$

$$
\begin{aligned}
\sigma^{2}(s, t)= & 2^{1-K}\left(\alpha^{2}+\beta^{2}\right)(t-s)^{2 H K} \\
& -(\alpha+\beta)^{2} F_{H, K}(s, t) \\
& +2^{1-K+2 H K} \alpha \beta F_{\frac{1}{2}, 2 H K}(s, t) .
\end{aligned}
$$

Proof. It suffices to combine (1.3), (3.1) and (3.2) with lemma 8.

The next step consists in determining the value of the constant $C_{2}$.

Combining Lemma 12 with Proposition 10, we have

if $0<2 H K<1$, then

$$
\begin{aligned}
\sigma^{2}(s, t) & \leq 2^{1-K}\left(\alpha^{2}+\beta^{2}\right)(t-s)^{2 H K} \\
& +2^{1-K+2 H K} \alpha \beta F_{\frac{1}{2}, 2 H K}(s, t),
\end{aligned}
$$


if $2 H K=1$, then

$$
\sigma^{2}(s, t) \leq 2^{1-K}\left(\alpha^{2}+\beta^{2}\right)(t-s)
$$

and

if $1<2 H K<2$, then

$$
\sigma^{2}(s, t) \leq 2^{1-K}\left(\alpha^{2}+\beta^{2}\right)(t-s)^{2 H K} .
$$

El-Nouty and Journé [12] showed that we have for $0<2 H K<1$,

$$
\begin{aligned}
(t-s)^{2 H K}+2^{2 H K-1} F_{\frac{1}{2}, 2 H K}(s, t) & \\
& \leq\left(2-2^{2 H K-1}\right)(t-s)^{2 H K} .
\end{aligned}
$$

Then,

$$
\begin{aligned}
\sigma^{2}(s, t) \leq & 2^{1-K}\left(\alpha^{2}+\beta^{2}\right)(t-s)^{2 H K} \\
& +2^{1-K+2 H K} \alpha \beta 2^{1-2 H K}\left(1-2^{2 H K-1}\right)(t-s)^{2 H K} \\
& =2^{1-K}\left((\alpha+\beta)^{2}-2^{2 H K} \alpha \beta\right)(t-s)^{2 H K} \\
& :=C_{2}(t-s)^{2 H K} .
\end{aligned}
$$

Let us determine the value of the constant $C_{1}$.

Combining Lemma 12 with Proposition 10, we get

$$
\begin{gathered}
\sigma^{2}(s, t) \geq 2^{1-K}\left(\alpha^{2}+\beta^{2}\right)(t-s)^{2 H K} \\
-(\alpha+\beta)^{2} F_{H, K}(s, t) .
\end{gathered}
$$

It was proved by El-Nouty and Journé [12] that, when $2 H K \leq 1$,

$$
\begin{aligned}
(t-s)^{2 H K}+2^{K}\left(t^{2 H K}\right. & \left.+s^{2 H K}\right)-2\left(t^{2 H}+s^{2 H}\right) \\
& \geq\left(2^{K}-1\right)(t-s)^{2 H K},
\end{aligned}
$$

that is

$$
\left(2-2^{K}\right)(t-s)^{2 H K} \geq 2^{K} F_{H, K}(s, t) .
$$

Then,

$$
\begin{aligned}
\sigma^{2}(s, t) & \geq\left((\alpha+\beta)^{2}-2^{2-K} \alpha \beta\right)(t-s)^{2 H K} \\
& :=C_{1}(t-s)^{2 H K} .
\end{aligned}
$$

Finally, we determine the value of the constant $C_{3}$. Recall that $s>0$. Set

$$
t-s=h .
$$

When $h \rightarrow 0$, the Taylor expansions of the functions $F_{H, K}$ and $F_{1 / 2,2 H K}$, given in (3.1) and (3.2), are

$$
\begin{aligned}
F_{H, K}(s, t)= & s^{2 H K}\left(H^{2} K(1-K) \frac{h^{2}}{s^{2}}\right. \\
& \left.+o\left(\frac{h^{2}}{s^{2}}\right)\right)
\end{aligned}
$$

and for $2 H K \neq 1$

$$
\begin{aligned}
F_{\frac{1}{2}, 2 H K}(s, t)= & -s^{2 H K}\left(\frac{H K(2 H K-1)}{2} \frac{h^{2}}{s^{2}}\right. \\
& \left.+o\left(\frac{h^{2}}{s^{2}}\right)\right) .
\end{aligned}
$$

Combining Lemma 12 with (3.3) and (3.4) (or Proposition 10 if $2 H K=1$ ), we obtain the Taylor expansion of $\sigma(s, t)$. Hence, since $2 H K<2$, we get the value of $C_{3}$.

To complete the proof of Theorem 6 , we have to verify that

$$
C_{1} \leq C_{3} \leq C_{2}
$$

and 


$$
C_{1} \leq C_{4} \leq C_{2} .
$$

Assume

$$
\alpha \geq \beta>0
$$

and set

$$
x=\frac{\alpha}{\beta}, x \geq 1
$$

The inequalities $C_{1} \leq C_{3} \leq C_{2}$ are equivalent to

$$
\begin{aligned}
x^{2}+\left(2-2^{2-K}\right) x+1 \\
\leq 2^{1-K}\left(x^{2}+1\right) \\
\leq 2^{1-K}\left(x^{2}+\left(2-2^{2 H K}\right) x+1\right),
\end{aligned}
$$

that is

$$
(x+1)^{2} \geq 0
$$

and

$$
\left(2-2^{2 H K}\right) x \geq 0
$$

Since $2 H K \leq 1$, it proves the result. Similarly, we can establish that

$$
C_{1} \leq C_{4} \leq C_{2}
$$

The proof of Theorem 6 is complete.

\section{REFERENCES}

1. Kahane J-P. Some random series of functions. Cambridge studies in advanced mathematics, Cambridge University Press, 1985.

2. Houdré C., Villa J. An Example of Infinite Dimensional Quasi-Helix. // Contemp. Math., 2003, Vol. 336, pp. 195-201

3. Bojdecki T., Gorostiza L.G., Talarczyk A. Sub-Fractional Brownian Motion and
Its Relation to Occupation Times. // Statist. Probab. Lett., 2004, Vol. 69, pp. 405-419.

4. El-Nouty C., Journé J-L., The subbifractional Brownian motion. // Studia Sci. Math. Hungar., 2013, Vol. 50, pp. 67-121.

5. Zili M., Generalized Fractional Brownian Motion. // Modern Stochastics: Theory and Applications, 2017, Vol. 4, pp. 15-24.

6. Berman S.M. Limit Theorems for the Maximum Term in Stationary Sequences. // Ann. Math. Statist, 1964, Vol. 35, pp. 502516.

7. Samorodnitsky G., Taqqu M.S. Stable non-Gaussian Random Processes. Chapman \& Hall, 1994.

8. El-Nouty C., Journé J-L. Upper Classes of the Bifractional Brownian Motion. // Studia Sci. Math. Hungar, 2011, Vol. 48, pp. 371-407.

9. El-Nouty C., On Approximately Stationary Gaussian Processes. // International Journal for Computational Civil and Structural Engineering, 2015, Vol. 11, pp. 15-26.

10. Bojdecki T., Gorostiza L.G., Talarczyk A. Some Extensions of Fractional Brownian Motion and Sub-Fractional Brownian Motion Related to Particle Systems. // Electron. Comm. Probab., 2007, Vol. 12, pp. 161-172.

11. El-Nouty C. The Increments of a Bifractional Brownian Motion. // Studia Sci. Math. Hungar., 2009, Vol. 46, pp. 449-478.

12. El-Nouty C., Journé J-L., The Subbifractional Brownian Motion. // Studia Sci. Math. Hungar, 2013, Vol. 50, pp. 67-121.

13. Russo F., Tudor C.A. On Bifractional Brownian Motion. // Stochastic Process. Appl., 2006, Vol. 116, pp. 830-856.

\section{СПИСОК ЛИТЕРАТУРЫ}

1. Kahane J-P. Some random series of functions. Cambridge studies in advanced mathematics, Cambridge University Press, 1985.

2. Houdré C., Villa J. An Example of Infinite Dimensional Quasi-Helix. // Contemp. Math., 
2003, Vol. 336, pp. 195-201

3. Bojdecki T., Gorostiza L.G., Talarczyk A. Sub-Fractional Brownian Motion and Its Relation to Occupation Times. // Statist. Probab. Lett., 2004, Vol. 69, pp. 405-419.

4. El-Nouty C., Journé J-L., The subbifractional Brownian motion. // Studia Sci. Math. Hungar., 2013, Vol. 50, pp. 67-121.

5. Zili M., Generalized Fractional Brownian Motion. // Modern Stochastics: Theory and Applications, 2017, Vol. 4, pp. 15-24.

6. Berman S.M. Limit Theorems for the Maximum Term in Stationary Sequences. // Ann. Math. Statist, 1964, Vol. 35, pp. 502516.

7. Samorodnitsky G., Taqqu M.S. Stable non-Gaussian Random Processes. Chapman \& Hall, 1994.

8. El-Nouty C., Journé J-L. Upper Classes of the Bifractional Brownian Motion. // Studia Sci. Math. Hungar, 2011, Vol. 48, pp. 371-407.

9. El-Nouty C., On Approximately Stationary Gaussian Processes. // International Journal for Computational Civil and Structural Engineering, 2015, Vol. 11, pp. 15-26.

10. Bojdecki T., Gorostiza L.G., Talarczyk A. Some Extensions of Fractional Brownian Motion and Sub-Fractional Brownian Motion Related to Particle Systems. // Electron. Comm. Probab., 2007, Vol. 12, pp. 161-172.

11. El-Nouty C. The Increments of a Bifractional Brownian Motion. // Studia Sci. Math. Hungar., 2009, Vol. 46, pp. 449-478.

12. El-Nouty C., Journé J-L., The Subbifractional Brownian Motion. // Studia Sci. Math. Hungar, 2013, Vol. 50, pp. 67-121.

13. Russo F., Tudor C.A. On Bifractional Brownian Motion. // Stochastic Process. Appl., 2006, Vol. 116, pp. 830-856.

Charles El-Nouty, Prof. Dr. hab., Université Paris XIII Sorbonne Paris Cité LAGA; 99 avenue J-B Clément, 93430 Villetaneuse;

E-mail: elnouty@math.univ-paris13.fr.
Шарль Эль-Нути, профессор, доктор математических наук, кафедра анализа, геометрии и прикладной математики, Университет Париж XIII, Сорбона - Париж Сите, Франция; 99 avenue J-B Clément, 93430 Villetaneuse, Франция;

E-mail: elnouty@math.univ-paris13.fr. 